\title{
Frontiers of Radiative Transfer
}

\author{
Thomas Henning \\ Astrophysical Institute and University Observatory (AIU), Friedrich \\ Schiller University, Schillergäßchen 3, D-07745 Jena, Germany
}

\begin{abstract}
Detailed radiative transfer calculations are an essential tool for the analysis of observations related to fragmented molecular cloud cores, protobinaries, and circumbinary and circumstellar disks. In this contribution, recent progress in line and continuum radiative transfer models will be reviewed.
\end{abstract}

\section{Introduction}

A thorough analysis of spectral energy distributions, line spectra, intensity, and polarization maps requires sophisticated numerical calculations of the photon transport. In addition, hydrodynamical simulations have to include radiative transfer to come to a realistic modeling of fragmentation processes in molecular clouds and the dynamics of accretion disks. In molecular cloud cores, dusty envelopes and disks, the radiation field is often far from a Planckian field. This means that the Eddington approximation, assuming that the radiation field is nearly isotropic, is not valid in these configurations.

The solution of the radiative transfer equation (see, e.g., Mihalas 1978) is often even more complicated than the solution of the hydrodynamical problem for the same configuration because the directional and frequency dependence of the radiation field introduces additional dimensions: for the time-independent 1-D case (slab or spherical symmetry) the specific intensity $\mathrm{I}_{\nu}(\mathrm{r}, \theta)$ is a function of three variables (frequency $\nu$, radial distance $r$, direction $\theta$ ). In the general 3 -D case, we have to consider $\mathrm{I}_{\nu}(\mathrm{x}, \mathrm{y}, \mathrm{z}, \theta, \phi)$ with the three spatial coordinates $x, y, z$ and the directional variables $\theta$ and $\phi$. An additional complication is the coupling between the source function $\mathrm{S}_{\nu}$ and the opacities with the intensity, and the shift of line frequencies due to turbulent or systematic motions in the case of line transfer.

Despite the various difficulties of numerical radiative transfer calculations, higher computer speed and storage capacity, the parallelizing of codes, and new numerical algorithms such as adaptive/nested grids (Steinacker \& Henning 1999, Wolf et al. 1999), accelerated convergence (Auer 1991), domain decomposition (Chick 1998), and generalization of the short characteristics method (Dullemond $\&$ Turolla 2000) led to a tremendous progress in recent years. Some of the new developments will be reviewed in this paper. 


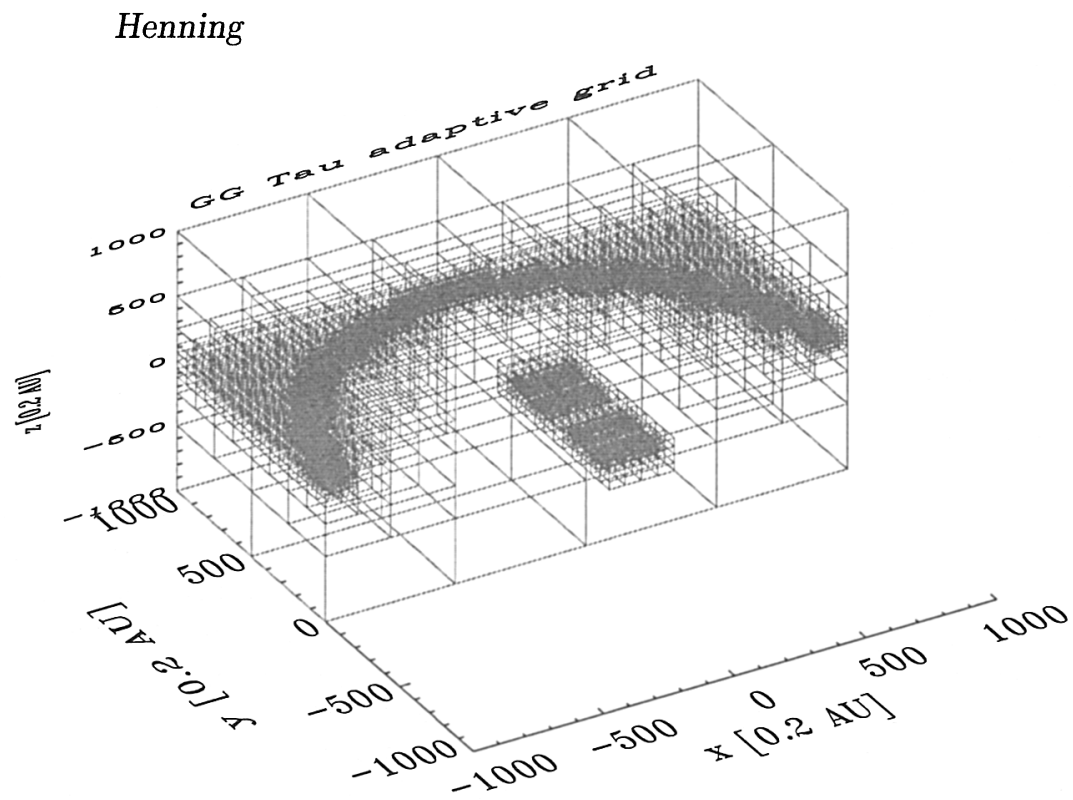

Figure 1. Adaptive grid for a circumbinary and two circumstellar disks - A grid construction appropriate for the GG Tau system. Figure provided by J. Steinacker.

\section{Radiative Transfer - More Dimensions}

A general goal of code development is the extension of the calculations to higher spatial dimensions. However, one should keep in mind that more complex configurations introduce additional parameters. This means that 2-D and 3-D calculations are only appropriate where the necessary information is provided by spatially-resolved observations or hydrodynamical models. Prominent examples are GG Tau with its circumbinary disk (Dutrey et al. 1994, Roddier et al. 1996, Silber et al. 2000) and hydrodynamical calculations of fragmentation and binary formation (Bodenheimer et al. 2000).

Similar to hydrodynamical calculations, we can distinguish grid-based and particle (Monte Carlo) methods. Monte Carlo codes are intrinsically 3D codes, although this feature is not always used. They are usually more flexible concerning the density structure and distribution of energy sources, and provide polarization maps. However, the error control is more difficult compared to grid-based codes.

1-D grid-based continuum radiative transfer calculations for spherical envelopes have been published in large number and are used routinely. The first computations have been performed more than 20 years ago (Leung 1976, Scoville \& Kwan 1976, Yorke 1977, Rowan-Robinson 1980). Deeply embedded single low-mass protostars and massive cocoon stars are examples of astrophysical objects where the application of spherically symmetry may be justified. It took another 10 years before the first 2-D calculations have been performed. Spagna et al. (1985) treated the radiative transfer in an externally heated disk with a quasi-diffusion method (see also Spagna et al. 1991). Efstathiou \& RowanRobinson (1990) presented results for flared disks assuming powerlaw densities 
in radius and azimuthal angle. Sonnhalter et al. (1995) studied several disk-halo structures with a flux-limited approximation. An approximate, but fast method has been designed by Menshchikov \& Henning (1997), assuming that the density only depends on the radial distance from the star. Based on an improved version of this code, Menshchikov et al. (1999) performed a detailed study of HL Tau.

There are only very few studies about 3-D continuum radiative transfer. A 3-D ray tracing code was outlined by Yorke (1986). Spectra and isophotes have been derived for single and binary protostellar clouds with 3-D frequencydependent calculations using temperature distributions obtained from either diffusion or Eddington approximation (Boss \& Yorke 1990). A 3-D code with adaptive grid construction has been developed by Steinacker (2000, see also Fig. 1). The first 3D Monte-Carlo code with self-consistent temperature treatment is now available (Wolf \& Henning 2000). It has been applied for protobinaries and disks by Wolf et al. (1999; see also the contribution by Wolf et al. in this volume).

Numerous 1-D line transfer codes applicable to molecular cloud cores are available (see, e.g., van Zadelhoff et al. 1999). 2-D codes with different degrees of sophistication have been developed by Hogerheijde et al. (1999) and Dullemond \& Turolla (2000). Juvela (1997) developed a 3-D code, but with limited spatial resolution.

\section{Radiative Transfer - More Physics}

The treatment of radiative transfer is always a combination of the structure of the configuration (heat sources, density distributions, clumpiness, velocity fields), and the microphysical properties of the medium.

In this section, we will only discuss the microphysical properties of grains. For the line transfer, we refer to a recent progress report by Ossenkopf (2000) who especially deals with the water lines. Continuum transfer calculations very often include one size and material only. This is clearly insufficient because any individual grain component will have their own temperature distribution. Averaging over material properties is a dangerous approximation (Boss \& Yorke 1996). The treatment of different grain sizes and materials became a standard in 1-D and even 2-D calculations (see, e.g., 1-D: Chini et al. 1986, Preibisch et al. 1993; 2-D: Menshchikov et al. 1999, Manske \& Henning 1999).

Many spectra of circumstellar disks and envelopes show evidence for emission from very small grains and PAHs. This requires the treatment of temperature fluctuations. There are only few codes available which include nonequlibrium heating (1D: Siebenmorgen et al. 1992, 2D: Manske \& Henning 1999). In optically thicker configurations multiple scattering is taken into account in many codes. However, the scattering is mostly treated as isotropic scattering (see, e.g. Rowan-Robinson 1980). Radiative transfer for configurations with (aligned) non-spherical grains and the treatment of the complete scattering process is a potential domain of Monte Carlo codes (Wolf et al. 2000). Finally, the temperature dependence of grain opacities (Henning \& Mutschke 1997) and their change with agglomeration state (Ossenkopf \& Henning 1994, Henning \& Stognienko 1996) has to be taken into account. Monte Carlo calculations provide simultaneously intensity and polarization maps - a feature which is extremely 


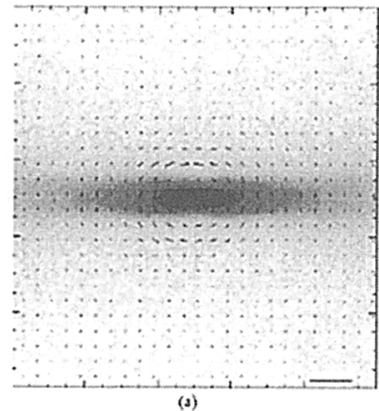

(נ)

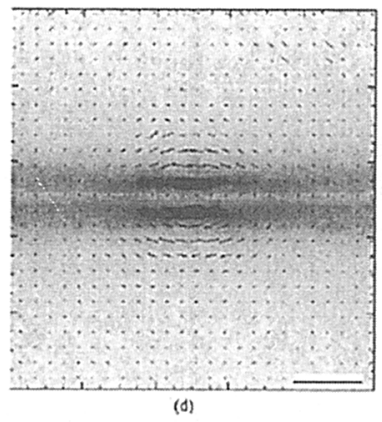

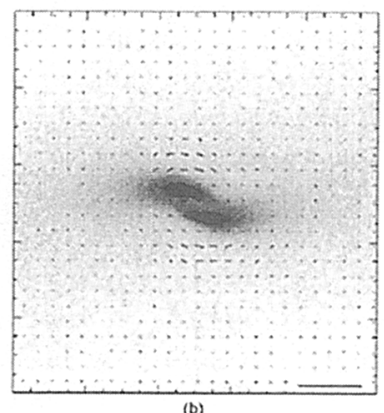

(b)

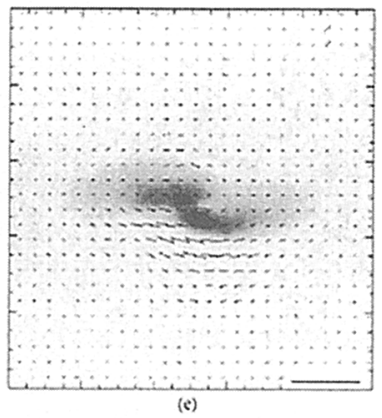

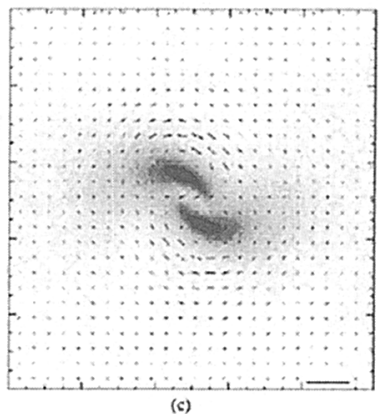

(c)

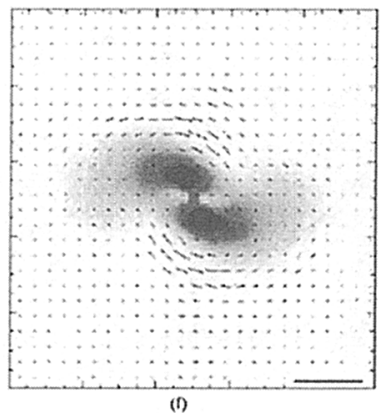

Figure 2. Intensity maps with overlayed polarization structures of a fragmented molecular cloud core. a-c: $\lambda=400 \mu \mathrm{m}$. d-f: $\lambda=90 \mu \mathrm{m}$. Diameter of the projected region: $237 \mathrm{AU}$. The vertical line in the lower right corner symbolizes the polarization degree of $6 \times 10^{-6}(\lambda=400 \mu \mathrm{m})$ and $4 \times 10^{-4}(\lambda=90 \mu \mathrm{m})$, produced by scattering. Inclination angles: a,d 90 degrees, b,e 60 degrees, c,f 0 degrees. Hydrodynamical model after Boss (1998). See Wolf et al. (1999) for details of the radiative transfer calculations.

helpful to constrain the parameter space. Here one should keep in mind that models may contain more variables/parameters than actually provided by the observations. This can easily lead to a non-uniqueness in the "derived" quantities (Thamm et al. 1994).

\section{Conclusions and Outlook}

Despite the progress in radiative transfer calculations for molecular clouds, protostars, and accretion disks, the lack of test cases for the often very complicated codes is a suprising fact. Even 1-D codes may fail to fulfill energy conservation if the photon transfer in configurations with very high optical depths has to be calculated. Benchmark problems for 1-D calculations can be found in Ivezic et al. (1997) (continuum transfer) and van Zadelhoff et al. (1999) (line trans- 
fer). The first test calculations for 2-D configurations have been performed by Menshchikov \& Henning (1997) and Wolf et al. (1999).

A goal for numerical radiative transfer will be to improve the speed of calculations with higher dimensions in order to couple radiative transfer with hydrodynamical models. Although first steps have been made for 3-D configurations (Klahr et al. 1999, Boss et al. 2000), this remains a programme for the next decade.

Acknowledgments. I acknowledge many discussion with my collaborators Alexander Menshchikov, Jürgen Steinacker and Sebastian Wolf. The size limitation of this paper did not allow to refer to all relevant papers. The reader will find additional references by reading the papers cited below.

\section{References}

Auer, L. H. 1991, in Stellar Atmospheres: Beyond Classical Models, ed. L. Crivellari et al. , Kluwer, Dordrecht, 9

Bodenheimer, P., Burkert, A., Klein, R. I., Boss, A. P. 2000, in Prostars and Planets IV, ed. V. Mannings, A. P. Boss, S. S. Russell, Univ. of Arizona Press, 675

Boss, A. P. 1998, ApJ, 501, L77

Boss, A. P., Yorke, H. W. 1990, ApJ, 353, 236

Boss, A. P., Yorke, H. W. 1996, ApJ, 469, 366

Boss, A. P., Fisher, R. T., Klein, R. I., McKee, C. F. 2000, ApJ, 528, 325

Chick, K. M. 1998, J. Quant. Spec. Rad. Transf., 59, 563

Chini, R., Krügel, E., Kreysa, E. 1986, A\&A, 167, 315

Dullemond, C. P., Turolla, R. 2000, A\&A, 360, 1187

Dutrey, A., Guilloteau, S., Simon, M. 1994, A\&A, 286, 149

Efstathiou, A., Rowan-Robinson, M. 1990, MNRAS, 245, 275

Henning, Th., Stognienko, R. 1996, A\&A, 311, 291

Henning, Th., Mutschke H. 1997, A\&A, 327, 743

Hogerheijde, M. R., van Dishoeck, E. F., Salverda, J. M., Blake, G. A. 1999, ApJ, 513, 350

Ivezic, Z., Groenewegen M. A. T., Menshchikov, A., Szczerba, R. 1997, MNRAS, 291,121

Juvela, M. 1997, A\&A, 322, 943

Klahr, H. H., Henning, Th., Kley, W. 1999, ApJ, 514, 325

Leung, C. M. 1976, ApJ, 209, 75

Manske, V., Henning, Th. 1999, A\&A, 349, 907

Menshchikov, A. B., Henning, Th. 1997, A\&A, 318, 879

Menshchikov, A. B., Henning, Th., Fischer, O. 1999, ApJ, 519, 257

Mihalas D. 1978, Stellar Atmospheres. 2nd, ed. W. H. Freeman, San Francisco

Ossenkopf, V. 2000, in ISO beyond the Peaks, ESA SP-456, in press

Ossenkopf, V., Henning, Th. 1994, A\&A, 291, 943. 
Preibisch, Th., Ossenkopf, V., Yorke, H. W., Henning, Th. 1993, A\&A, 279, 577

Roddier C., Roddier, F., Northcott, M. J., Graves, J. E., Jim, K. 1996, ApJ, 463,326

Rowan-Robinson, M. 1980, ApJS, 44, 403

Scoville N. Z., Kwan J. 1976, ApJS, 206, 718

Siebenmorgen, R., Krügel E., Mathis, J. S. 1992, A\&A, 266, 501

Silber, J., Gledhill, T., Duchêne, G., Ménard, F. 2000, ApJ, 536, 89

Sonnhalter, C., Preibisch, Th., Yorke, H. W. 1995, A\&A, 299, 545

Spagna, G. F., Leung, C. M., Egan, M. P. 1985, Icarus, 61, 27

Spagna, G. F., Leung, C. M., Egan, M. P. 1991, ApJ, 379, 232

Steinacker, J. 2000, in preparation

Steinacker, J., Henning, Th. 1999, in Optical and Infrared Spectroscopy, ed. E.

W. Guenther, B. Stecklum, S. Klose, ASP Conf. Ser., 188, 109

Thamm, E., Steinacker, J., Henning, Th. 1994, A\&A, 287, 493

Wolf, S., Henning, Th., Stecklum, B. 1999, A\&A, 349, 839

Wolf, S., Henning, Th. 2000, Comp. Phys. Comm., 132, 166

Wolf, S., Voshchinnikov, N., Henning, Th. 2000, in preparation

Yorke, H. W. 1977, A\&A, 58, 423

Yorke, H. W. 1986, in Astrophyscial Radiation Hydrodynamics, ed. K.-H.A. Winkler, M. L. Norman, Reidel, Dordrecht, 141

van Zadelhoff et al. 1999, http://www.strw.LeidenUniv.nl/ radtrans/

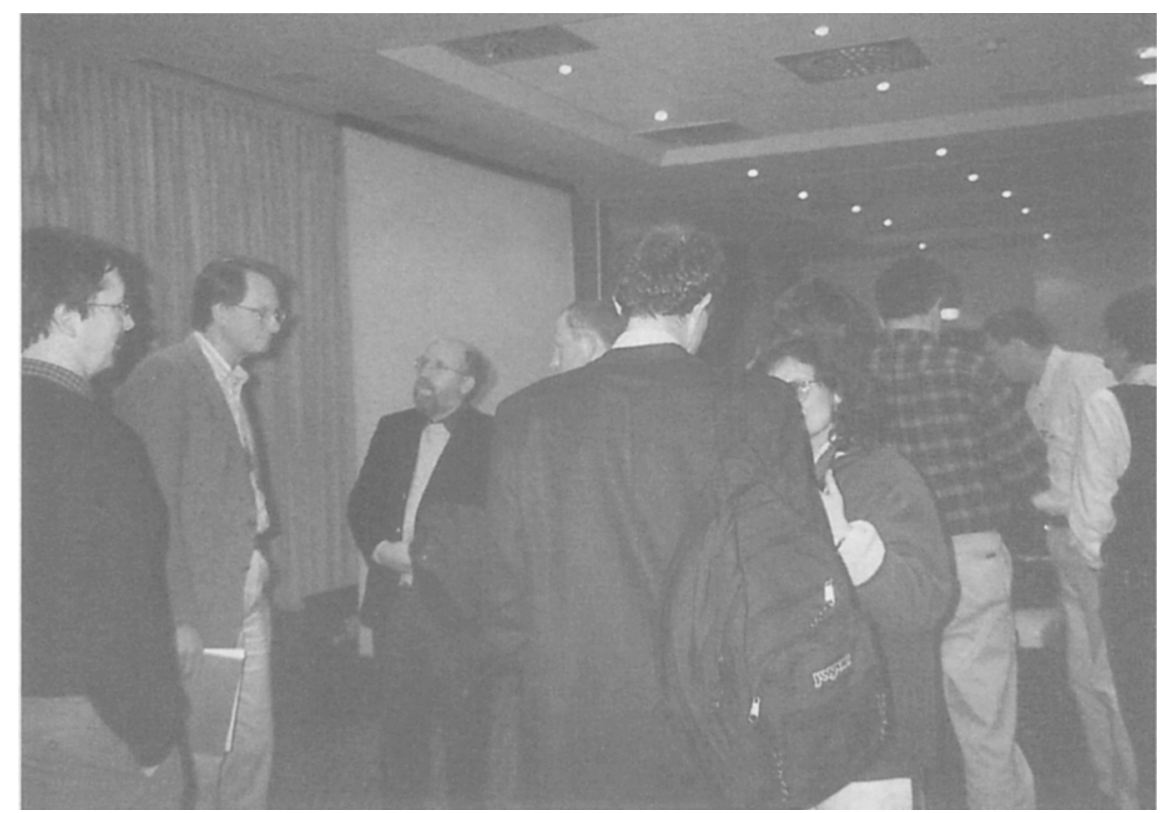

Thomas Henning, Alan Boss, Michel Mayor et al. 